\title{
Exposure to Secondhand Smoke Exacerbated Natural Aging Cardiac Hypertrophy
}

\author{
Jia-Ping $\mathrm{Wu}^{*}$
}

Research Center for Healthcare Industry Innovation, National Taipei University of Nursing and Health Sciences, Taiwan

ISSN: 2578-0204

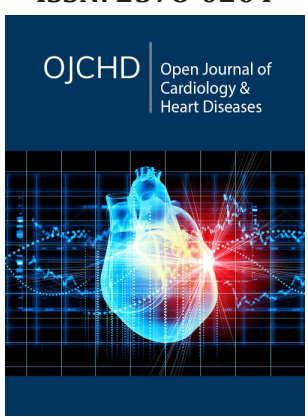

*Corresponding author: Jia-Ping $\mathrm{Wu}$, Research Center for Healthcare Industry Innovation, National Taipei University of Nursing and Health Sciences, Taiwan

Submission: 眮 October 22, 2019

Published: 眥January 17, 2020

Volume 3 - Issue 2

How to cite this article: Jia-Ping Wu. Exposure to Secondhand Smoke Exacerbated Natural Aging Cardiac Hypertrophy. Open J Cardiol Heart Dis.3(2). OJCHD.000557.2020.

DOI: 10.31031/OJCHD.2020.03.000557.

Copyright@ Jia-Ping Wu, This article is distributed under the terms of the Creative Commons Attribution 4.0 International License, which permits unrestricted use and redistribution provided that the original author and source are credited.

\section{Abstracts}

Secondhand Smoke (SHS) exposure is associated with an increased risk of coronary artery disease. This study's aim was to investigate the relationship of SHS exposure in old rats' left ventricle impaired and fibrosis. To explore the mechanism of cardiac remodeling of exposure to SHS exposure whether it was exacerbated cardiac impaired, especially in the older left ventricle. The animals were placed in a transparent exposure chamber, connected to smoking device and exposed to 15 cigarettes, smoke for $30 \mathrm{~min}$, twice a day, 6 days/week, for 1 month. Histopathologic of left ventricular sections were stained with hematoxylin-eosin staining (H\&E) and Masson's trichrome. Left Ventricular (LV) morphological variables assessed using H\&E stained and Mass weight changes. The cardiac structures were measured by echocardiographic analysis.

LV remodeling and fibrosis-related proteins were detected by gelatin zymography and western blotting analysis. Inflammatory and hypertrophy related proteins were also detected. Results showed in old rats' group and old rats in the secondhand smoke exposure group (Old SHS Exp) were observed LV wall and mass increased, collagen accumulation and fibrosis, and extracellular space increased. From echocardiographic results, we found LV functions were apparently decreased, LV interventricular septum at systolic and diastolic diameters increased in the Old SHS Exp group. Cardiomyocyte width was increased in old rats, but the length was increased in Old SHS Exp group. Reduced MMP 2 proteins expression and TIMPs increased were induced fibrosis in the Old SHS Exp group. JNK1, p38, IL-6, TNF $\alpha$ were increased by western blotting and immunohistochemistry antibody-positive expression was observed in the Old SHS Exp group.

Keywords: Secondhand smoke exposure; Left ventricle; Cardiac impaired; Left ventricular hypertrophy; Echocardiographic; Cardiomyocyte

\section{Introduction}

Secondhand smoke (SHS) exposure is a serious health hazard causing the risk of coronary heart diseases. It causes a wide range of damaging health effects in children and older. SHS exposure affected on the cardiovascular system, including atherosclerosis, arterial stiffness and coronary cardiac disease [1]. In the previous studies reported, SHS exposure is the combination of smoke given off by the burred end of a tobacco or cigarette product to exposure to environment and the smoke exhaled by the smoker [2]. However, low-level chronic cigarette smoke exposure harmful older adults are still unclear. In this study, we use the older rats who underwent 15 cigarette exposure 30 minutes to determined left ventricular remodeling and function. Hypertrophy is an initial adaptive response. Long-term exposure to secondhand smoke increases the risk of developing cancer in younger and elderly people [3].

Low levels of fine particulate exposure from secondhand cigarette smoke are sufficient to induce increase the risk of cardiovascular disease mortality. The SHS exposure-response relationship between cardiovascular disease mortality and fine particulate matter is relatively steep at low levels of SHS exposure and flattens at higher exposures [4]. There are many compensatory mechanisms to increase cardiac workload and stimulation of left ventricular sustains. However, aging is a progressive disease which is a typic natural course whose worsening of the disease until death occurs. Slowly progressive age-related diseases are also chronic diseases; many are also degenerative diseases [5].

The aging mature organism that occurs normally the gradual changes in the structure over time and increases the probability of death. This growing process is unavoidable. 
These physiologic changes of old cardiac include left ventricular hypertrophy, increased cardiac fibrosis, and valvular degeneration. Cardiovascular disease is a major risk factor for the aging cause of death [6]. Aging changes in the elderly heart are associated with physiological Left Ventricular Hypertrophy (LVH). However, SHS exposure is associated with pathological LVH [7]. SHS exposure in the elderly maybe leads to cardiovascular diseases such as heart failure and atherosclerosis. SHS exposure in the old heart is still unclear. Heart failure is a related change in cardiac morphology, including decreased in myocyte number, increased in myocytes size decreased in matrix connective tissue, increased in left ventricular wall thickness increased in conduction fiber density and decreased in sinus node cell number [8-10]. SHS exposure in the elderly may stimuli first induce a phase of cardiac hypertrophy, especially in left ventricles individual. Health aging changes may produce clinical heart disease and may mimic heart diseases, such as cardiomyopathy, aortic valve calcium and mitral valve annular calcium [11,12]. Therefore, we detected the molecular mechanisms behind the aging in SHS exposure treatment to identify pathological of cardiac disease disorder and elusive.

\section{Materials and Methods}

\section{Animals}

We purchased SD rats of 6 weeks years-old age from National Science Council Animal Center and used according to the guidelines of the Helsinki Declaration. One group of rats of 6-weeks-old rats as our young, another group of rats of 18-months-old as our older age groups. Rats were housed in cages in an environmentally controlled animal room. Use committee approved animal care and experiments. Animal room temperature is maintained at $25^{\circ} \mathrm{C}$, and relative humidity was approximately $40 \%$.

\section{Secondhand Smoke (SHS) exposure experimental}

The elderly SD rats placed in a whole-body transparent exposure chamber with a volume of approximately $95 \times 85 \times 85 \mathrm{~cm}$, connected to a smoking device. Filtered air is introduced into the chamber at a low rate. Puffs of SHS exposure were collected in the smoking chamber, is then thrown into the chamber for 30 minutes. The smoke is released at a rate of 15 cigarettes, twice a day in the morning and twice in the afternoon with 30 minutes rest intervals, until the end of 4 weeks.

\section{Echocardiography}

After 4 weeks of exposure treatment, all the rats underwent echocardiographic study according to the previously described method. Rats used anesthetized with ketamine hydrochloride $(50 \mathrm{mg} / \mathrm{kg})$ and xylazine hydrochloride $(1 \mathrm{mg} / \mathrm{kg})$. Transthoracic echocardiography was performed at 4 weeks after secondhand smoke (SHS) exposure using a Hewlett-Packard Sonos 5500 ultrasound machine with a 7.5-15 $\mathrm{MHz}$ linear-array transducer, as described previously. In the short-and long- axis parasternal view, we could obtain a transverse left ventricular one-dimensional image, the ultrasound beam right below the mitral valve plane between the papillary muscles by using the 2D image as a guide for positioning. The M-mode image was recorded and analyzed offline.

\section{Hematoxylin-eosin (H\&E) and massons trichrome (mt) stained}

Left ventricular cross-sections were cut $10 \mu \mathrm{m}$ thick and placed on slides. Slides deparaffinization and dehydration were performed. They were passed through a series of graded alcohols from $100 \%$ to $90 \%$ to $70 \%$, 5min each. Hematoxylin-eosin and Masson trichome stained were prepared, incubated for $5 \mathrm{~min}$ at room temperature. After rinsing with Phosphate-Buffered Saline (PBS), each slide was then soaked with $85 \%$ alcohol, $100 \%$ alcohol for $5 \mathrm{~min}$. After rinsing with water, each slide was then soaked with $85 \%$ alcohol, $100 \%$ alcohol for $15 \mathrm{~min}$. Stained sections were then rinsed with PBS and air-dried before mounting.

\section{Gelatin zymography}

Proteins were separated by $8 \%$ non-reducing SDS-PAGE copolymerized with $1 \mathrm{mg} / \mathrm{ml}$ gelatin. The PAGE was washed at room temperature twice 10 minutes with $2.5 \%$ Triton-X 100 and subsequently incubated overnight at $37^{\circ} \mathrm{C}$ for maximum sensitivity in Zymogram Developing Buffer mixture $(50 \mathrm{mmol} / \mathrm{L}$ Tris- $\mathrm{HCl}$, pH 7.4 containing $5 \mathrm{mmol} / \mathrm{L} \mathrm{CaCl}_{2}$ and $1 \mu \mathrm{mol} / \mathrm{L} \mathrm{ZnCl}_{2}$ ). Gels were stained with Coomassie brilliant blue G250 (Methanol, Acetic acid, and water mix) and then destained. The amounts of proenzyme and active metalloproteinase were analyzed by densitometry scanning of the gel.

\section{Western blot}

We prepared the tissue extract samples as described above. SDS-PAGE was carried out with polyacrylamide gels. The samples were electrophoresed at $100 \mathrm{~V}$ for $1 \mathrm{hr}$. Electrophoresed proteins were transferred to PVDF membranes at $150 \mathrm{~mA}$ for $2 \mathrm{hr}$. We incubated PVDF membranes in blocking buffer (5\% non-fat milk in PBS-Tween) for $1 \mathrm{hr}$ at room temperature. Polyclonal antibodies against JNK1/2, p38 $\alpha$, IL-6, TNF $\alpha$, MMP2, MMP9, TIMP-1, TIMP-2, TIMP-3 and TIMP-4 (Santa Cruz, Dallas, Texas, U.S.A.) were diluted 1:200 in antibody buffer (TBS). Incubations were performed at room temperature for $3.5 \mathrm{hr}$. We washed the immunoblots three times in $5 \mathrm{ml}$ PBS-Tween for $10 \mathrm{~min}$ and then immersed in the second antibody solution containing alkaline phosphatase goat anti-rabbit IgG for $1 \mathrm{hr}$ and diluted 1,000-fold in binding buffer. Color development was presented in ECL chemiluminescence.

\section{Ethical Statement}

Animals guidelines for the animal experimental use of Taipei Medical University Animal Care and Use Committee (IACUC) (LAC2019-0264) and ARRIVE Guidelines were followed. The Taiwan Council approved the animal care and experiment. All procedures followed have been performed in accordance with the ethical standards laid down in the 1964 Declaration of Helsinki and its later amendments.

\section{Statistical Analysis}

Quantitation was carried out by scanning and analyzing the intensity of the hybridization signals using the FUJIFILM Imagine program for western blot analysis. Statistical analysis of the data was performed using Sigma Stat software. Results were expressed 
as mean \pm SEM. Statistical analysis was performed using the analysis of variance. When assessing multiple groups, one-way ANOVA was utilized with the students t-test was used when indicated.

\section{Result}

Histopathologic of a left ventricular cross-sectional analysis assessed cardiac changes in old rats in cigarette smoke exposure by H\&E stained and masson's stained. To investigate the effects of secondhand smoke (SHS) exposure on cardiac functions and structural changes were determined in rats model recommended for gerontological. Heart cross-sections were stained with Massonss trichrome or hematoxylin/eosin staining for visualization of morphology and identification of the location. A cross-sectional analysis assessed left ventricular changes in old rats and old rats in the SHS exposure group. As shown in Figure 1A. left ventricular chamber becomes narrowed in old rats in the SHS exposure group (Old SHS Exp).

(A)
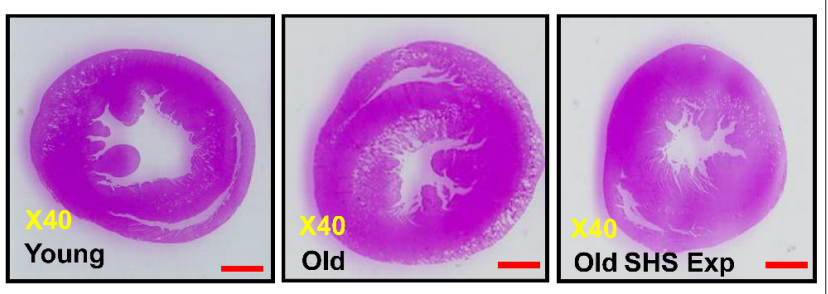

(B)
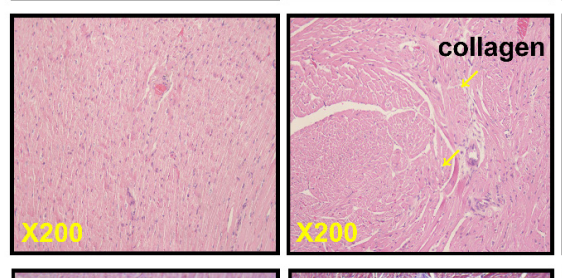

(C)
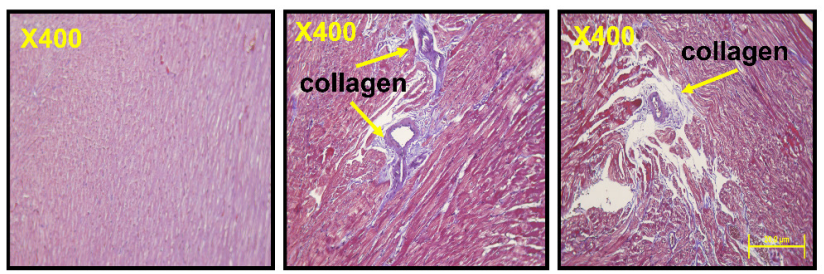

Figure 1: Representative histopathological analysis of left ventricular cross-sections with hematoxylin $\&$ eosin (H\&E) and Masson's trichrome staining in young, old rats and old rats in the SHS exposure groups (Old SHS Exp).

(A) Representative by hematoxylin \& eosin staining of left ventricular sections in young, old and old SHS Exp groups

(B) Representative collagen accumulation in the left ventricle by hematoxylin $\&$ eosin staining in young, old and old SHS Exp groups. Scale bars $20 \mu \mathrm{m}$. The images of left ventricular architectures were magnified 200x. Yellow arrows express

(C) Representative collagen accumulation in the left ventricle by Masson's trichrome staining in young, old and old SHS Exp groups. Scale bars $20 \mu \mathrm{m}$. The images of left ventricular architectures were magnified 400x. Yellow arrows express.

At the same time, SHS exposure resulted in old rats' left ventricular papillary muscle deteriorated which led to left ventricular dysfunction. Left ventricular muscle fibers interstitial and extracellular space was broad. Muscle fibers' rearrangement is disordered. In old rats and old rats in the SHS exposure group, we also could observe ECM degradation resulted in collagen release in cardiomyocytes interstitial (Figure 1B) and collagen accumulation induced fibrosis. Indeed, from Masson's trichrome stained results, we could observe blue color staining in cross-sections (Figure 1C).

\section{Changes in structures development of heart in old rats and old rats in SHS exposure group}

Figure 2 presents heart and Left Ventricular (LV) characteristics in young, old and old rats in the SHS exposure (Old SHS Exp). The whole heart weights of old rats and old rats in the SHS exposure were heavier than young rats. Aging and SHS exposure rats were also enhanced left ventricular weights $\left({ }^{*} \mathrm{p}<0.05\right.$ vs. young rats; $\# \mathrm{p}<0.05$ vs. old rats) (Figure $2 \mathrm{~A}$ ). The HW-to-body weight and LVto-body weight ratios were significantly increased compared with younger age group. Compared with old rats, the HW-to-body weight and LV-to-body weight ratios also had significantly increased (Figure $2 \mathrm{~B}$ ) in Old SHS Exp groups ( ${ }^{*} \mathrm{p}<0.05$ vs. young rats; $\# \mathrm{p}<0.05$ vs. old rats). However, body weight is easily caused by the gradual increase in a consequence of aging. Sometimes affected by alcohol, smoke or toxic material effects. The use of tibia length has been evidenced as more reliable than body weight. Because of in which body weight differences may occur condition errors. 


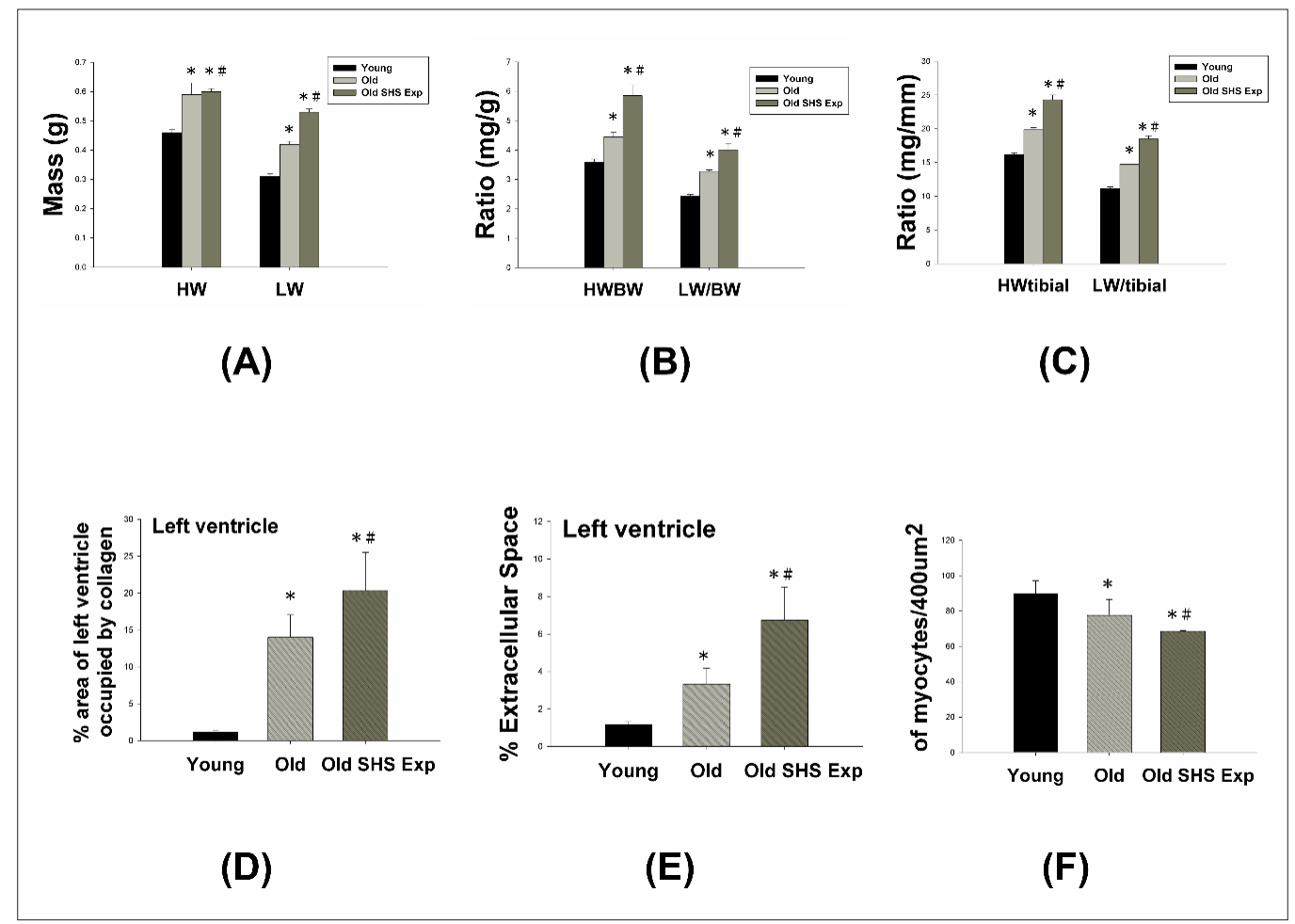

Figure 2: Left ventricular hypertrophy takes place in old rats and old rats in the SHS exposure group (Old SHS Exp).

(A) Quantification of heart weight and left ventricle weight statistical analysis. All data are represented as the means \pm SEM. ${ }^{*} \mathrm{p}<0.05$ compared with young rats. $\# \mathrm{p}<0.05$ compared with old rats

(B) Quantification of the heart weight to body ratio and the ratio of left ventricular weight to body weight statistical analysis. All data are represented as the means \pm SEM. ${ }^{*} \mathrm{p}<0.05$ compared with young rats. \#p $<0.05$ compared with old rats

(C) Quantification of heart weight to tibial ratio and the ratio of left ventricular weight to tibial statistical analysis. All data are represented as the means \pm SEM. ${ }^{*} \mathrm{p}<0.05$ compared with young rats. $\# \mathrm{p}<0.05$ compared with old rats

(D) Quantification of percent of area of left ventricle occupied by collage. All data are represented as the means \pm SEM. * $\mathrm{p}<0.05$ compared with young rats. $\# \mathrm{p}<0.05$ compared with old rats

(E) Quantification of percent (\%) left ventricle extramyocyte connective tissue space (area). All data are represented as the means \pm SEM. ${ }^{*} p<0.05$ compared with young rats. $\# p<0.05$ compared with old rats $(\mathrm{F})$. Quantification of average number of myocytes per $400 \mu \mathrm{m}$. Values were calculated from myocardial regions. All data are represented as the means \pm SEM. ${ }^{*} \mathrm{p}<0.05$ compared with young rats. $\# \mathrm{p}<0.05$ compared with old rats.

Furthermore, we detected and measured HW-to-tibial and LVto-tibial ratios to determine heart or left ventricular hypertrophy in old rats and to exposed to SHS exposure (Old SHS Exp). The result showed both of HW-to-tibial and LV-to-tibial ratios were increased significantly as age increased, although exposure to SHS exposure (Figure $3 C$ ) $\left({ }^{*} \mathrm{p}<0.05\right.$ vs. young rats; $\# \mathrm{p}<0.05$ vs. old rats). Therefore, we obtained HW-to-tibial and LV-to-tibial ratios were increased compared with old rats. To detect whether SHS exposure led left ventricular fibrosis exacerbated, we independently calculated the percentage of the per cross-sectional area from hematoxylinstained sections. Quantification of the percentage of the area of left ventricle occupied by collagen (\%), collagen area was measured in old rats and old rats in SHS exposure group (Old SHS Exp). The percentage of tissue attributed to collagen distribution increased more rapidly in old rats in the SHS exposure group than in old or young rats ( ${ }^{*} \mathrm{p}<0.05$ vs. young rats; $\# \mathrm{p}<0.05$ vs. old rats) (Figure 3 ). To detect whether SHS exposure led left ventricular hypertrophy exacerbated, we independently calculated the percentage of monocytes per cross-sectional area from hematoxylin-stained sections.

Quantification of left ventricular muscle fibers interstitial width of extracellular space, we found left ventricular muscle fibers interstitial become broad resulted in the percentage of extracellular space (\%) increased $\left({ }^{*} \mathrm{p}<0.05\right.$ vs. young rats; $\# \mathrm{p}<0.05$ vs. old rats) (Figure 3). During old age or exposure to SHS exposure, left ventricles from old rats and old rats in the SHS Exp group exhibited fewer percentage per unit area than young rats $\left({ }^{*} \mathrm{p}<0.05\right.$ vs. young rats; $\# p<0.05$ vs. old rats) (Figure 3). But cardiomyocytes density is increased per unit area than young rats (result does not 
show in this article). Indeed, according to tissue architecture using H\&E staining analysis, we determined cell size measurement. We observed left ventricular cell size increased width in old rats.

\section{Left ventricular function and structures development of left ventricle on echocardiographic analysis}

The echocardiographic analysis is a primary imaging method in the assessment of cardiac impaired and function declined (Figure 3). Parasternal long-axis and short-axis echocardiographic views in young, old and old rats in the SHS exposure (Old SHS Exp) groups showing severe left ventricular hypertrophy. We found left ventricular wall thickness increased. However, M-mode echocardiograms result taken proximal to the papillary muscle deterioration in old rats and old rats in the SHS exposure group (Figure 3A). On the other hand, we found left ventricular wall thickness increases in old rats and old rats in the SHS exposure at systolic and diastolic. Quantification of myocardial hypertrophy for old rats and old rats in the SHS exposure groups are displayed in Figure 3B. Echocardiography of interventricular septal in systolic (IVSs), interventricular septal in diastolic (IVSd), left ventricular internal dimension at end-systolic (LVIDs), left ventricular internal dimension at end-diastolic (LVIDd), left ventricular posterior wall thickness in systolic (LVPWs) and left ventricular posterior wall thickness in diastolic (LVPWd) were presented in young, old and old rats in the SHS exposure group.
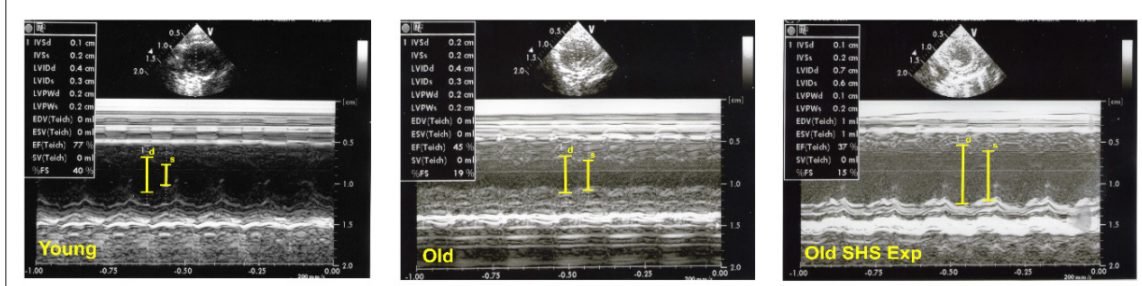

(A)

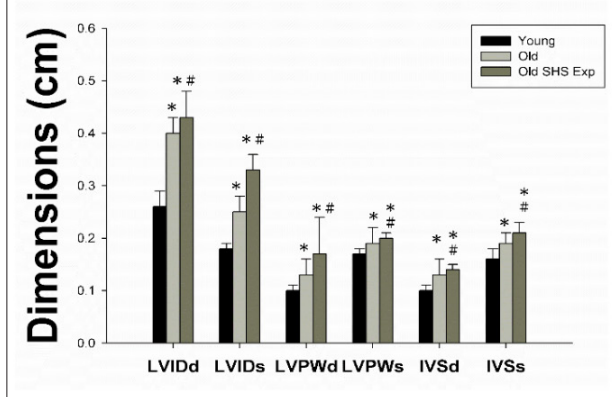

(B)

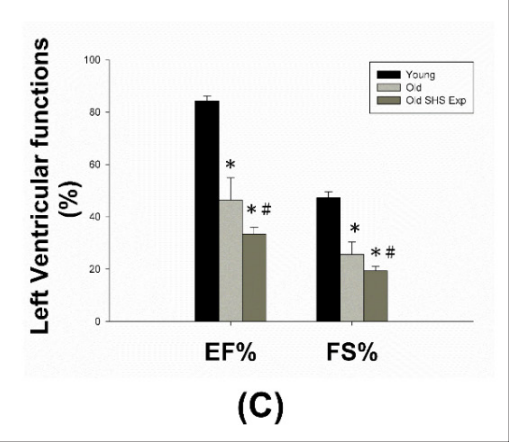

Figure 3: Histopathologic of left ventricular hypertrophy using M-mode echocardiograms in young, old rats and old rats in the SHS exposure group (Old SHS Exp)

(A) Representative M-mode echocardiograms taken proximal from young, old and old SHS Exp group. These images were obtained from short-and long-axis imaging at the mid-papillary level. Parasternal short-axis echocardiography views (up-panel), parasternal long-axis echocardiography views (down-panel)

(B) Quantification of left ventricular posterior wall thickness at diastolic and systolic, interventricular septal in systolic (IVSs), interventricular septal in diastolic (IVSd), left ventricular internal dimension at end systolic (LVIDs), left ventricular internal dimension at end diastolic (LVIDd), left ventricular posterior wall thickness in systolic (LVPWs) and left ventricular posterior wall thickness in diastolic (LVPWd) shown in right panel. All data are represented as the means \pm SEM. *p $<0.05$ compared with young rats. $\#$ p $<0.05$ compared with old rats

(C) Quantification of the percentage of fractional shorting and ejection fraction at diastolic and systolic. All data are represented as the means \pm SEM. ${ }^{*} \mathrm{p}<0.05$ compared with young rats. $\# \mathrm{p}<0.05$ compared with old rats.

The morphological variables obtained from the echocardiographic study are shown in Figure 3. The old rats in SHS exposure had greater IVSs, IVSd, LVIDs, LVIDd, LVPWs and LVPWd dimension compared with young rats $\left({ }^{*} \mathrm{p}<0.05\right.$ vs. young rats; $\# \mathrm{p}<0.05$ vs. old rats). After exposure to SHS, the old rats had statistically greater dimensions than nonsmoking old rats did. This variable change was used to confirm the efficacy of the exposure of old rats to secondhand smoke (SHS). In addition, considering the left ventricular variables, the ejection and shortening fractions were significantly declined. As Figure 3C shown, shortening (FS\%) and ejection fraction (EF\%) displayed a progressive impairment in old rats and old rats in the SHS exposure group (Old SHS Exp) $\left({ }^{*} \mathrm{p}<0.05\right.$ vs. young rats; $\# \mathrm{p}<0.05$ vs. old rats). 
Cardiomyocytes width and length of left ventricular hypertrophy in old rats and old rats in the SHS exposure group

The phase of left ventricular hypertrophy during adaptive stress or overload is individual left ventricular myocyte grown in length and/or width as compensated or dilation hypertrophy (Figure 4A).
According to the left ventricular cell width size, old rats in the SHS exposure compared with old rats were increased $\left({ }^{*} p<0.05\right.$ vs. young rats). According to compared with left ventricular cell length size in old rats and old rats in the SHS exposure group was increased (Figure 4B). Compared with old rats, left ventricular cell length size in old rats in the SHS exposure group was significantly increased $\left({ }^{*} \mathrm{p}<0.05\right.$ vs. young rats; $\# \mathrm{p}<0.05$ vs. old rats $)$.

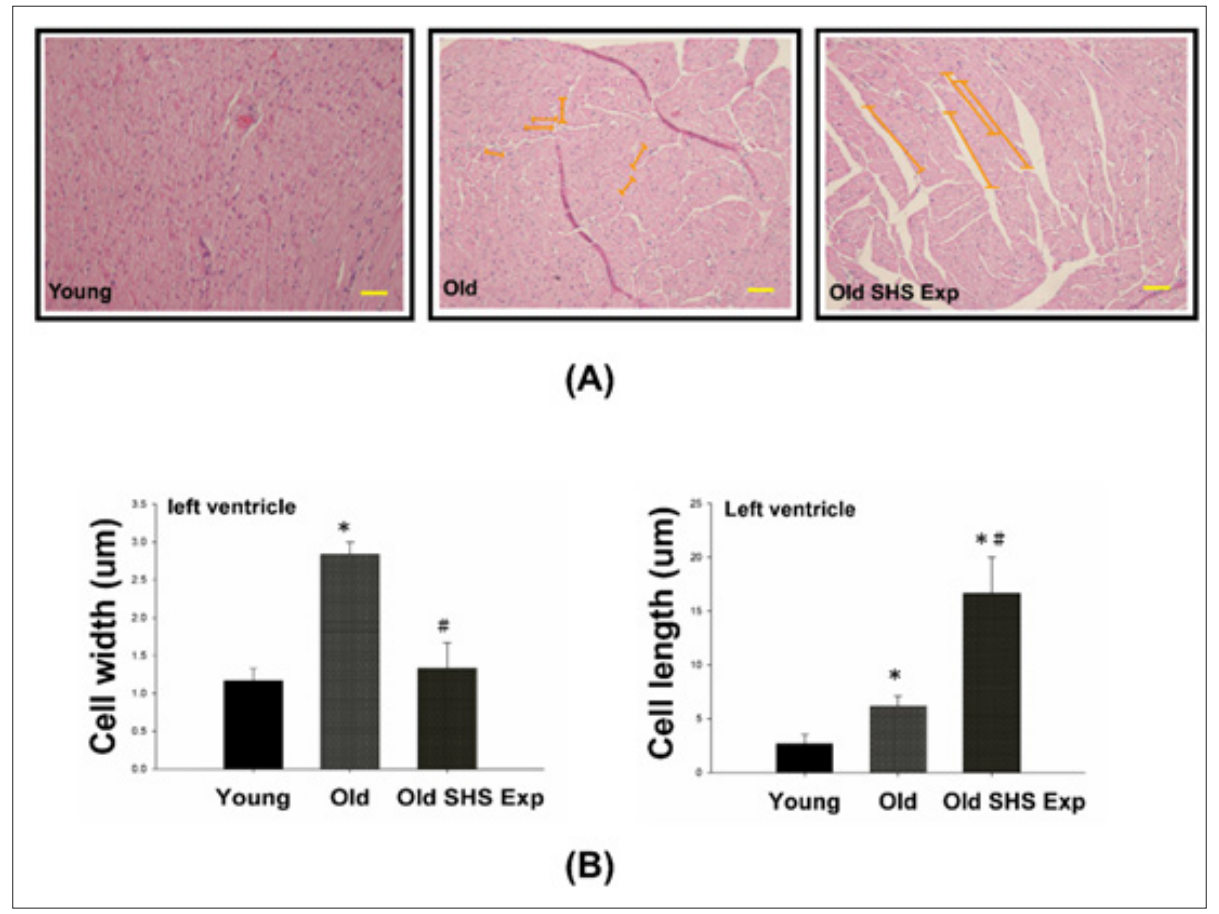

Figure 4: Longer cardiomyocytes occur left ventricular hypertrophy in the SHS exposure group by hematoxylin $\&$ eosin stained.

(A) Representative long cardiomyocytes occur left ventricular hypertrophy using histological sections by $\mathrm{H} \& \mathrm{E}$ stained to determine cell width $(\mu \mathrm{m})$ and cell length $(\mu \mathrm{m})$ in young, old rats and old rats in the SHS exposure group (Old SHS Exp)

(B) Quantification of cell width $(\mu \mathrm{m})$ and cell length $(\mu \mathrm{m})$ in young, old rats and old rats in the SHS exposure group (Old SHS Exp). Values are represented as the means \pm SEM. * $\mathrm{p}<0.05$ compared with young rats. \#p $<0.05$ compared with old rats.

\section{Changes to MMPs' protein expression can explain age- related heart failure disease}

Fibrosis occurs from changes in the balance between synthesis and degradation of extracellular matrix components. Therefore, we sought to determine whether aging and SHS exposure-related collagen accumulation and fibrosis could be related to changes in the regulation of MMP2 and MMP9. Gelatin zymography detected 2 major gelatinolytic bands, MMP-2 and MMP-9, in the left ventricular extracts. MMP-9 gelatinolytic bands did not observe. As Figure 5A shown. Because its regulation was the sum of pro-MMP-9 and TIMP1, it may be MMP-9 complexed with TIMP-1. The down regulation of MMP-2 in old rats and old rats in the SHS exposure group was statistically significant compared with young rats. The extent of changes in MMP-2 gelatinolytic activity was lower than old rats $\left({ }^{*} \mathrm{p}<0.05\right.$ vs. young rats; $\# \mathrm{p}<0.05$ vs. old rats). Consistent with the results of gelatin zymography, MMP-2 and MMP-9 protein content as measured by western blotting was also significantly reduced in old rats and old rats in the SHS exposure group (Old SHS Exp) (Figure 5B).

These results suggest that MMP2/MMP9 contributed to the remodeling of the extracellular matrix in left ventricular fibrosis. While aging, we found MMP2 and MMP9 protein expression decreased. Once exposure to SHS exposure, MMP2, and MMP9 protein expression were significantly lower than old rats $\left({ }^{*} \mathrm{p}<0.05\right.$ vs. young rats; $\# \mathrm{p}<0.05$ vs. old rats). The dysregulation of MMP2/ MMP9 was now believed to contribute to fibrosis in old and SHS exposure. Elevated TIMPs expression induced fibrosis is present in old rats and old rats in SHS exposure. MMPs catalyze ECM degradation, TIMPs is physiological inhibitors which controlled MMPs activity. 


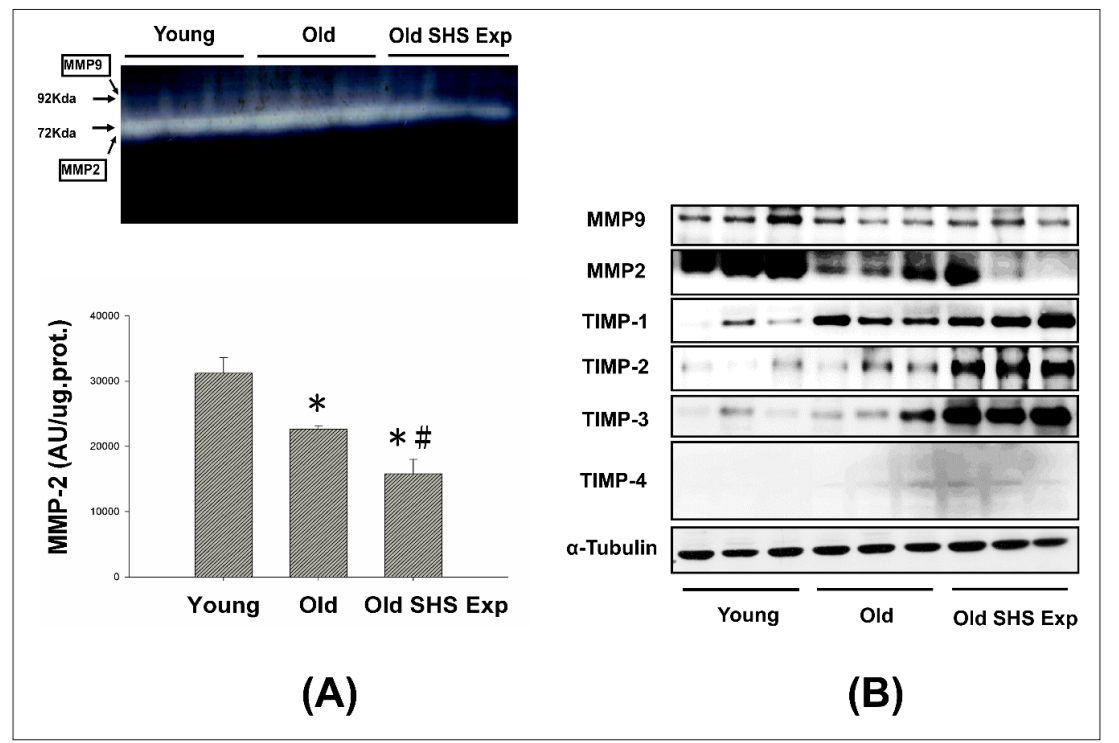

Figure 5: Molecular mechanisms of the imbalance of MMPs and TIMPs induced ECM remodeling.

(A) Representative zymographical analysis from young, old rats and old rats in the SHS exposure group (Old SHS Exp). Up-panel shows a gelatin zymography graphic representative of MMP-2 and MMP-9 activity. Down-panel shows MMP-2 activity quantified by densitometry and expressed as mean pixel density. Values are represented as the means \pm SEM. ${ }^{*} \mathrm{p}<0.05$ compared with young rats. $\# \mathrm{p}<0.05$ compared with old rats

(B) Protein expression activity dysregulation of MMP-2, MMP-9 and protein expression elevated of TIMP-1, TIMP2, TIMP-3 and TIMP-4 in old rats and old rats in the SHS exposure group (Old SHS Exp) by western blotting.

To explore whether there are regulation changes in the expression of TIMPs, we assessed the protein expression of TIMPs (TIMP-1, $-2,-3$ and -4 ) by Western blot. An up regulation of TIMPs is associated with heart failure and fibrosis. To determine whether aging or SHS exposure modulates cardiac matrix remodeling. As Figure 5B shown, we found TIMP-1, TIMP-2, TIMP-3 and TIMP4 induced left ventricular fibrosis, protein expression levels by western blotting analysis in old rats and old rats in the SHS exposure group were significantly higher than young rats. Thus, this apparent cause of fibrosis and heart failure can be explained by differential regulation between MMPs and TIMPs.

\section{SHS exposure results in higher sensitivity to inflammation and heart failure in old rats}

SHS exposure is an environmental stressor induced pathological LVH. In this study, we determine whether low concentration SHS exposure to old rats also will be induced pathological LVH. Results showed p-MEK5/MEK5 and p-ERK5/ERK5 increased in old rats in the SHS exposure group (Old SHS Exp), but not in the old hearts (Figure 6A). To further determine the potential of inflammation in the old hearts, we examined JNK1/2, p38 $\alpha$, IL-6, and TNF $\alpha$ protein expression levels by western blotting and immunohistochemistry. As Figure 6B and Figure 6C shown, JNK1/2, p38 $\alpha$, IL-6, and TNF $\alpha$ protein expression levels were increased in old rats and old rats in the SHS exposure group (Old SHS Exp). Immunohistochemical study showed that densities of both old rats and old rats in the SHS exposure hearts were higher in young rats (Figure 6C). IL-6 and $\mathrm{TNF} \alpha$ play an important role in promoting LVH and inflammation. With greater age comes, we found that MAPK (JNK1/2 and p38) protein expression were increased in old rats and rats in the SHS exposure group. SHS exposure may enhance proinflammatory cytokines (IL-6 and TNF $\alpha$ ) and MAPK cascade expression in old rat hearts. Thus, cytokines in left ventricular hypertrophy tissues increased markedly in keeping with a denser inflammatory cell infiltration.

\section{Discussion}

Secondhand Smoke (SHS) exposure has been linked to a number of harmful health outcomes and is an important cause of morbidity and mortality. SHS exposure is an important cause of morbidity and mortality. There is a lot of evidence indicated that SHS exposure a formidable health hazard. However, there is no evidence indicated that SHS exposure presents a challenging health hazard [13]. It is also well understood that toxic air contamination causes lung cancer and cardiovascular diseases. In this study, we investigated the effects of SHS exposure associated with the elderly age, specifically in the left ventricles of male rats. As is well known, old age in humans always accompanies an increased incidence of atherosclerotic vascular disease and cardiovascular disease [14]. In contrast, aging is a physiological process due to increasing injuries and vulnerability, which reduces the ability of organisms to survive. Aging affects various aspects of left ventricular morphology and function and has recently been considered to be a major risk factor for cardiovascular disease and to have effects on various aspects of left vascular morphology and function. Aging can refer to a time-related process, however, it is commonly used for postmaturational processes. The main characteristics associated with aging is a progressive decrease in physiological capacities [15]. 


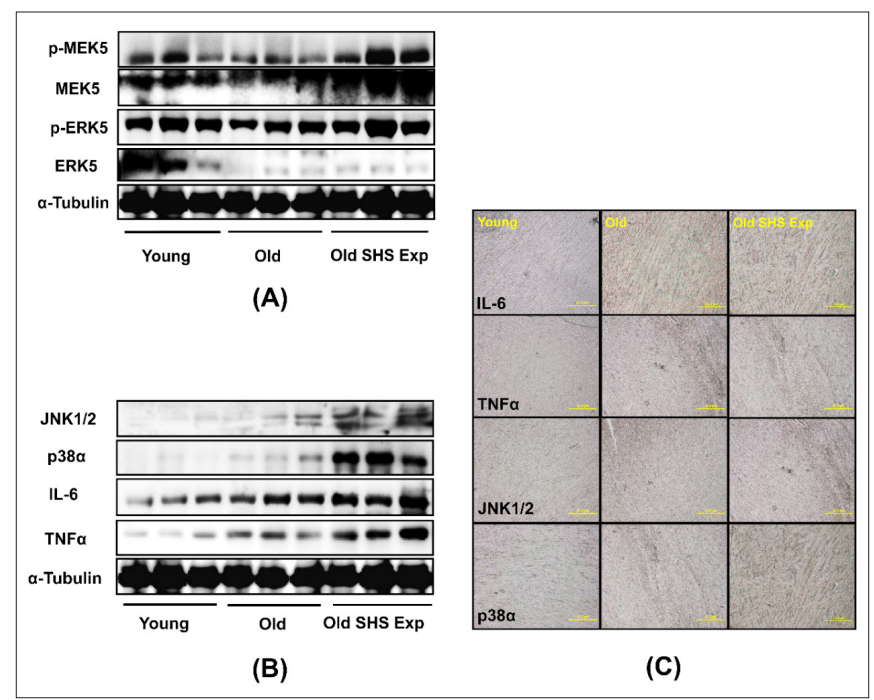

Figure 6: The effects of SHS exposure on the activation of molecular mechanisms of inflammation and left ventricular hypertrophy during aging.

(A) Upregulation of left ventricular hypertrophy proteins, p-MEK5/MEK5 and p-ERK5/ERK5 in old rats in the SHS exposure group by western blot

(B) Upregulation of the expression of inflammatory mediators, cytokines (IL-6 and TNFa) and MAPKs (JNK1/2 and $\mathrm{p} 38$ ), which lead to inflammation response

(C) Immunohistochemistry of IL-6, TNFa, JNK and p38 in young, old rats and old rats in SHS exposure groups.

The overall effect is highly debatable aging and disease. As age increases, whether there will occur diseases itself. However, the most obvious evidence of changes in the aging heart and liver. Biological phenomena appear related to the aging process [16]. Aging exacerbates cardiac damage, leading to cardiac hypertrophy, fibrosis [17] and dysfunction, developed compensatory concentric hypertrophy [18] and fibrosis in response to induced cardiomyocytes hypertrophy in a similar manner [19]. In the aging heart, demonstrated severe left ventricular chamber dilation, wall thinning and

fibrosis, leading to congestive heart failure. In this study, we want to know low-level chronic cigarette smoke exposure whether is harmful to left ventricular function in old rats and to explore the related mechanisms. We found that changes associated with SHS exposure lead to cardiovascular pathological outcomes resulted in age-related disease exacerbated. We observed left ventricular chamber narrowing and rupture and increased left ventricular wall thickness. These results demonstrated left ventricular hypertrophy in old rats and old rats in the SHS exposed group (Old SHS Exp). On the other hand, we could from echocardiography results to determine left ventricular dimension, posterior wall thickness, interventricular septal at end-systole and end-diastole were increased, and left ventricular function declined. Stiffening of these fibers cause left ventricular fibrosis and could also affect the efficient functioning. SHS exposure is linked to a number of harmful health outcomes.

As is well known, SHS exposure is a key risk factor for pathological hypertrophy associated with various cardiovascular disease risk factors [20], however, the old annual human always accompanies with atherosclerotic vascular disease and cardiovascular disease. As the heart reaches senescence, it undergoes a modest degree of Heart failure [21]. It is now determined the differences several signaling molecules play unique role in regulation of old rats in the SHS exposure group. We discuss molecular signaling mechanisms associated with old rats in the SHS exposure, including MMPs, TIMPs, JNK1/2, p38 $\alpha$, IL-6 and TNF $\alpha$ signaling. We suggested that upregulation of pro-inflammatory related protein expression of JNK1/2, p38 $\alpha$, IL-6 and TNF $\alpha$ enhanced left ventricular pathological hypertrophy. Down-regulation of MMP2 and MMP9 in old rats in the SHS exposure accelerated TIMPs-induced cardiac fibrosis. Despite the evidences that chronic exposure to SHS exposure resulted in cardiac changes [22,23], the exact mechanisms involved in lowlevel concentration process remain to be elucidated. These explore knowledge may influence therapeutic strategies for the treatment of cardiovascular disease in old age.

\section{Acknowledgment}

The study was approved by National Taipei University of Nursing and Health Sciences. No extra-institutional funding must be reported for this article.

\section{References}

1. Spychala MS, Venna VR, Jandzinski M, Doran SJ, Durgan DJ, et al. (2018) Age-related changes in the gut microbiota influence systemic inflammation and stroke outcome. Ann Neurol 84(1): 23-36.

2. Stout MB, Justice JN, Nicklas BJ, Kirkland JL (2017) Physiological aging: Links among adipose tissue dysfunction, diabetes, and frailty. Physiology 32(1): 9-19. 
3. Liu P, Xu B, Cavalieri TA, Hock CE (2002) Age-related difference in myocardial function and inflammation in a rat model of myocardial ischemia-reperfusion. Cardiovasc Res 56(3): 443-453.

4. Zhu J, Rebecchi MJ, Glass PS, Brink PR, Liu L, et al. (2011) Cardio protection of the aged rat heart by GSK-3beta inhibitor is attenuated: Age-related changes in mitochondrial permeability transition pore modulation. Am J Physiol Heart Circ Physiol 300(3): H922-H930.

5. Jiang Z, Rebecchi MJ, Qiang W, Glass PSA (2013) Chronic tempol treatment restores pharmacological preconditioning in the senescent rat heart. Am J Physiol Heart Circ Physiol 304(5): H649-H659.

6. Dinas PC, Koutedakis Y, Flouris AD (2013) Effects of active and passive tobacco cigarette smoking on heart rate variability. International Journal of Cardiology 163(2): 109-115.

7. Flouris AD, Vardavas CI, Metsios GS, Tsatsakis AM, Koutedakis Y, et al. (2010) Biological evidence for the acute health effects of secondhand smoke exposure. Am J Physiol Lung Cell Mol Physiol 298(1): L3-L12.

8. Brink TC, Demetrius L, Lehrach H, Adjaye J (2009) Age-related transcriptional changes in gene expression in different organs of mice support the metabolic stability theory of aging. Biogerontology 10(5): 549-564.

9. Garvin AM, Jackson MA, Korzick DH (2018) Inhibition of programmed necrosis limits infarct size through altered mitochondrial and immune responses in the aged female rat heart. Am J Physiol Heart Circ Physiol 315(5): H1434-H1442.

10. Liu P, Xu B, Cavalieri TA, Hock CE (2002) Age-related difference in myocardial function and inflammation in a rat model of myocardial ischemia-reperfusion. Cardiovasc Res 56(3): 443-453

11. Juonala M, Magnussen CG, Venn A, Gall S, Kähönen M, et al. (2012) Parental smoking in childhood and brachial artery flow-mediated dilatation in young adults: The cardiovascular risk in young finns study and the childhood determinants of adult health study. Arterioscler Thromb Vasc Biol 32(4): 1024-1031.

12. Kizaki K, Momozaki M, Akatsuka K, Fujimori Y, Uchide T, et al. (2004) Impaired gene expression of beta 1-adrenergic receptor, but not stimulatory G-protein Gs alpha, in rat ventricular myocardium treated with isoproterenol. Biol Pharm Bull 27(7): 1130-1132.
13. Kelly G (2010) A review of the sirtuin system, its clinical implications, and the potential role of dietary activators like resveratrol: Part 1. Altern Med Rev 15(3): 245-263.

14. Bard RL, Dvonch JT, Kaciroti N, Lustig SA, Brook RD, et al. (2010) Is acute high-dose secondhand smoke exposure always harmful to microvascular function in healthy adults? Prev Cardiol 13(4): 175-189.

15.Zhu L, Di PY, Wu R, Pinkerton KE, Chen Y, et al. (2015) Repression of CC16 by cigarette smoke (CS) exposure. PLoS One 10(1): e0116159.

16. Koutros S, Silverman DT, Alavanja MCR, Andreotti G, Lerro CC, et al. (2016) Occupational exposure to pesticides and bladder cancer risk. Int J Epidemiol 45(3): 792-805

17. Yang Z, Ming XF (2012) mTOR signalling: The molecular interface connecting metabolic stress, aging and cardiovascular diseases. Obesity Reviews 13: 58-68.

18. de Almeida AJPO, Ribeiro TP, de Medeiros IA (2017) Aging: Molecular pathways and implications on the cardiovascular system. Oxid Med Cell Longev p. 7941563.

19. Venn A, Britton J (2007) Exposure to secondhand smoke and biomarkers of cardiovascular disease risk in never-smoking adults. Circulation 115(8): 990-995.

20. Sheydina A, Riordon DR, Boheler KR (2011) Molecular mechanisms of cardiomyocyte aging. Clin Sci 121(8): 315-329.

21. Ungvari Z, Csiszar A (2012) The emerging role of IGF-1 deficiency in cardiovascular aging: Recent advances. Gerontol A Biol Sci Med Sci 67(6): 599-610.

22. Gielen S, Sandri M, Kozarez I, Kratzsch J, Teupser D, et al. (2012) Exercise training attenuates MuRF-1 expression in the skeletal muscle of patients with chronic heart failure independent of age: The randomized leipzig exercise intervention in chronic heart failure and aging catabolism study. Circulation 125(22): 2716-2727.

23. Alagiyawanna AMAAP, Veerasingam EQ Townsend N (2018) Prevalence and correlates of exposure to secondhand smoke (SHS) among 14 to 15-year-old schoolchildren in a medical officer of health area in Sri Lanka. BMC Public Health 18(1): 1240. 